\title{
The thermal visualisation of latent fingermarks on metallic surfaces
}

\author{
G. Wightman *, D. O'Connor \\ School of Contemporary Sciences, University of Abertay Dundee, Bell Street, Dundee DD1 1HG, Scotland, United Kingdom
}

A R T I C L E I N F O

\section{Article history:}

Received 14 September 2009

Received in revised form 13 May 2010

Accepted 17 May 2010

Available online xxx

\section{Keywords:}

Forensic science

Latent fingerprints

Latent fingermarks

Metal

Metallic surfaces

Enhancement

Visualisation

\begin{abstract}
A B S T R A C T
Recent published research has lead to improved techniques for recovering latent fingermarks from metallic surfaces. The present study corroborates and extends some of the work carried out by Bond [13], but an alternative mechanism is proposed for the thermal visualisation of fingermarks based on differential oxidation and the production of interference colours that improve contrast. Fingermarks treated at low temperature could be reheated to enhance recovery, but an upper temperature limit occurs beyond which the mark degrades. The mechanism of enhancement is discussed.
\end{abstract}

(c) 2010 Elsevier Ireland Ltd. All rights reserved.

\section{Introduction}

Research continues into the ability to develop latent fingermarks on metal. Fingermarks can be recovered by a range of traditional methods such as powdering and cyanoacrylate fuming but these sometimes have limitations and a range of alternative techniques for mark enhancement have been considered over the years. A significant development was made with the recent work published by Bond [1-3], and in these papers he reviews earlier research into fingermarks on metals.

In his initial study Bond [1] examined 40 fingermarks that had been stored at ambient temperature and normal humidity. In the second part of the study, 360 depositions were made on metal samples. These were heated using a propane gas burner to simulate exposure to a fire, and development of the mark sometimes occurred. The effect of humidity was investigated by treating 40 half-marks immediately and treating the other half-mark after a period of storage in a desiccator, and the degree of development was found to be independent of the length of time stored in the absence of humidity. In a third part of this study electrostatic enhancement was employed on 80 depositions with a typical potential of $2.5 \mathrm{kV}$ applied to the metal and a conducting powder applied which adhered to regions of the mark. This work was extended [2] by looking at the corrosion produced by mark depositions on 10 different metals and 10 alloys stored in air at

\footnotetext{
* Corresponding author. Tel.: +44 1382 308659; fax: +44 1382308104 .

E-mail address: g.wightman@abertay.ac.uk (G. Wightman).
}

ambient temperature. 40 donors were employed for each metal to allow for natural variations in the composition of body secretions. From these experimental observations and from thermodynamic considerations, a fingermark corrosion series was produced.

From these studies Bond concluded that development of the mark is due to the chloride ion present in perspiration from eccrine glands. Bond [1] suggested that the mechanism is initiated by a corrosion reaction:

$$
\begin{aligned}
& \mathrm{M} \rightarrow \mathrm{M}^{z+}+z \mathrm{e}^{-} \\
& 2 \mathrm{e}^{-}+\mathrm{H}_{2} \mathrm{O}+0.5 \mathrm{O}_{2} \rightarrow 2 \mathrm{OH}^{-}
\end{aligned}
$$

Normally these ions combine and form $\mathrm{M}(\mathrm{OH})_{z}$. However, Bond notes that "under certain conditions, the current density at the anode can result in the formation of a localised large concentration of metal ions that attract negatively charged ions and, for chloride ions, leads to the formation of hydrochloric acid in an autocatalytic reaction

$\mathrm{M}^{z+}+z \mathrm{Cl}^{-}+z \mathrm{H}_{2} \mathrm{O} \rightarrow \mathrm{M}(\mathrm{OH})_{z}+z\left(\mathrm{H}^{+}+\mathrm{Cl}^{-}\right)$

In the localised area, the $\mathrm{pH}$ of the solution decreases and the concentration of the $\mathrm{Cl}^{-}$ion increases".

Thermodynamically this reaction will normally go in the reverse direction (acid plus metal hydroxide forming salt plus water) unless it is driven by the activities of the reagents. Chloride ion is known to induce pitting corrosion in various metals and several mechanisms have been proposed based on chloride ions causing a breakdown in the protective oxide layer on the metal [4]. 
According to Bond [1], when metal is heated heat-induced corrosion occurs, with organic components of perspiration being destroyed and the inorganic salts reacting with the metal substrate and this corrosion enhances the latent mark. Corrosion products have increased resistivity compared with the metal and this results in a lower potential above the corrosion product when applying a voltage to the metal, and this in turn affects the attraction of the powder. The electrostatic technique proved unsuccessful on aluminium and this would be consistent with the high resistivity of the oxide layer on aluminium. One possible question about this mechanism is whether the enhancement is by chloride ions producing an insulating layer, or whether chloride ions are substituting in the oxide layer and increasing its conductivity.

Our present study was undertaken to investigate the thermal recovery of fingermarks. Not only will this help in understanding the recovery of fingermarks where materials have been subjected to heat such as in fire scenes, but also it may offer another tool when other enhancement techniques have proved unsuccessful. However, thermal treatment always needs to be considered as part of a hierarchy of evidence examination since heat can degrade other evidence that may be present. In this study it soon became apparent that the role of the oxide film formed on the metal was important in terms of the contrast achieved in the developed mark. The present study also raises questions as to the role of electrochemical corrosion in the high temperature treatment of fingermarks.

\section{Methodology}

Virgin metal discs were laser-cut from brass, aluminium and stainless steel. The protective polymer film was removed and the surface cleaned with acetone. Three donors were selected and, prior to each mark donation, they washed their hands with mild detergent and dried them on a paper towel. A 30-min period was allowed for secretions to build up on their hands, and during this period the donors avoided activities that might lead to excessive perspiration or to contamination of the mark. After 30 min, a mark was collected and the disc was left in the air for 5 min before being stored in a cushioned wallet for storing compact discs (CDs). Discs were stored for periods varying from $3 \mathrm{~h}$ to 7 days before thermal treatment.

Bond [1] used a propane gas burner and thermocouple to simulate the effect of metal being heated in a fire. In the present study thermal treatment was carried out in a Carbolite AAF 1100 furnace which can be rapidly heated due to its low thermal mass, and provides a stable temperature and unchanging atmosphere. Once the furnace had reached the required initial temperature $\left(200{ }^{\circ} \mathrm{C}\right.$ or $600{ }^{\circ} \mathrm{C}$ depending on the metal), the three donor discs plus a blank disc were placed on a ceramic boat and inserted in the furnace for $5 \mathrm{~min}$. On removal, the discs were allowed to cool in air for $20 \mathrm{~min}$ before being examined and photographed. The furnace was set to the next temperature (which was $200^{\circ} \mathrm{C}$ or $150{ }^{\circ} \mathrm{C}$ higher) and the procedure repeated. The same discs were reheated for a third time at a third temperature.

A second series of tests was also carried out with fresh discs and with a different set of temperatures to the first series in order to extend the range of conditions. The temperatures employed were chosen depending on the metal, and conditions are given in Table 1.

The fingermarks obtained were graded using a simplified version of Bond's [1] grading with 4 rather than 5 grades:

0 : no visualisation of a fingermark

1: very small amount of dot-like shapes, but no definite detail

2: visualisation of dots and slight identification of ridges

3: definite visualisation of ridges

Table 1

Test conditions.

\begin{tabular}{llll}
\hline & \multicolumn{2}{l}{ Metal } & \\
\cline { 2 - 4 } & Brass & Aluminium & Stainless steel \\
\hline Temperature ${ }^{\circ} \mathrm{C}$ & 200 & 200 & 600 \\
& 400 & 400 & 750 \\
600 & 600 & 900 \\
& 180 & 180 & 380 \\
280 & 280 & 530 \\
& 380 & 380 & 680
\end{tabular}

Number of donors: 3. Elapsed time before analysis: 3 h, 1 day, 3 days, 5 days, and 7 days.
Several of the fingermarks were observed under an optical microscope.

From the early observations of the fingermark development it was thought that secretions in the fingermark may be inhibiting oxide growth, and in order to test this hypothesis a disc of brass and a disc of stainless steel were both contacted with a range of chemicals: saline solution, solid sodium chloride, graphite, paraffin oil, and oleic acid with a fingermark included as a control for comparison with previous tests. These discs were heated sequentially at $200^{\circ} \mathrm{C}, 400{ }^{\circ} \mathrm{C}, 600^{\circ} \mathrm{C}$, and $750{ }^{\circ} \mathrm{C}$ following the same procedure as for the fingermarks and any evidence of oxidation was observed. Sodium chloride was selected to investigate whether it produced corrosion or inhibited oxidation. The three carbon-based materials were included to examine whether oxide reduction may occur.

\section{Discussion of results}

\subsection{Presentation of results}

Ensuring reproducibility of results, particularly when using human subjects, can be difficult. In the present study a protocol was adopted to try and minimise variations. All metal samples were cut to the same size, although the thickness of the three different metals varied slightly. Using a furnace rather than a flame ensures a more even heat rate and a constant temperature. A flame may be more representative of the conditions experienced in a fire or firearms discharge, but a furnace treatment is more suitable for laboratory work and offers greater control for mark recovery. Ensuring the latent mark was as consistent as possible was achieved by having a standard protocol for the donors. Despite this, there will be natural variations, for example due to emotional state, ambient temperature or the type of activity taking place during the donation period.

The quality of the marks for each of the 270 sets of conditions was reviewed, and the effect of each variable was examined and displayed as a graph. For each data set (e.g. one donor on one metal and one storage time) there will be a limited number of data points for the indeterminate variable (e.g. six temperatures). The use of a 4-point scale for the determinate variable also results in a coarse gradation since the interpretation of any individual mark could vary by 1 unit. However, examination of these individual graphs helped identify any anomalies and the photographs of the marks were then reviewed. A cumulative grade was calculated where all the individual mark grades within a set of results for one chosen variable were totalled. For example, to consider the effect of temperature on mark quality the grades from all three donors and all five storage times were combined. This approach reduces the weighting of each individual point and proved to be a reasonable way of quantifying and comparing the data. Since the set of secondary variables are the same for each primary variable, their effects should be similar across the range of conditions and hence should cancel out.

The results are shown in Figs. 1 and 2 and in Tables 2-5.

\subsection{Variation between donors}

Table 2 shows the cumulative grading of fingermarks for each donor with a maximum grading of 90 , and Table 3 shows the number of marks observed at each grade with a maximum value of 30 . Over the range of temperatures examined $\left(180-600{ }^{\circ} \mathrm{C}\right.$ for aluminium and brass, and $380-900{ }^{\circ} \mathrm{C}$ for stainless steel) and times ( $3 \mathrm{~h}$ to 7 days) certain trends can be observed. Donor 3 consistently gave better marks on all three metals and donor 1 was marginally better than donor 2, mainly due to the grade 3 marks achieved on brass but there is little difference between the two donors on the other two metals. Donor 3 also gave reasonable recovery on aluminium ( 21 out of 30 marks graded as 2 or 3 ).

Looking at individual results, donors 1 and 2 appeared to give poorer results with brass at low temperatures $\left(180^{\circ} \mathrm{C}\right.$ or $\left.200^{\circ} \mathrm{C}\right)$ after $3 \mathrm{~h}$ storage, but the mark improved at higher temperatures $\left(380^{\circ} \mathrm{C}\right)$, whereas donor 3 gave consistently good marks on brass 
(a)

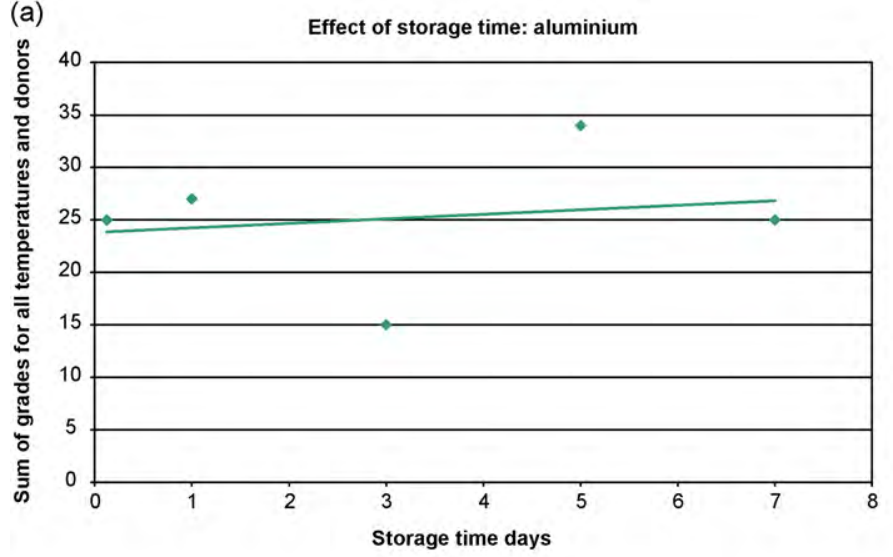

(b)

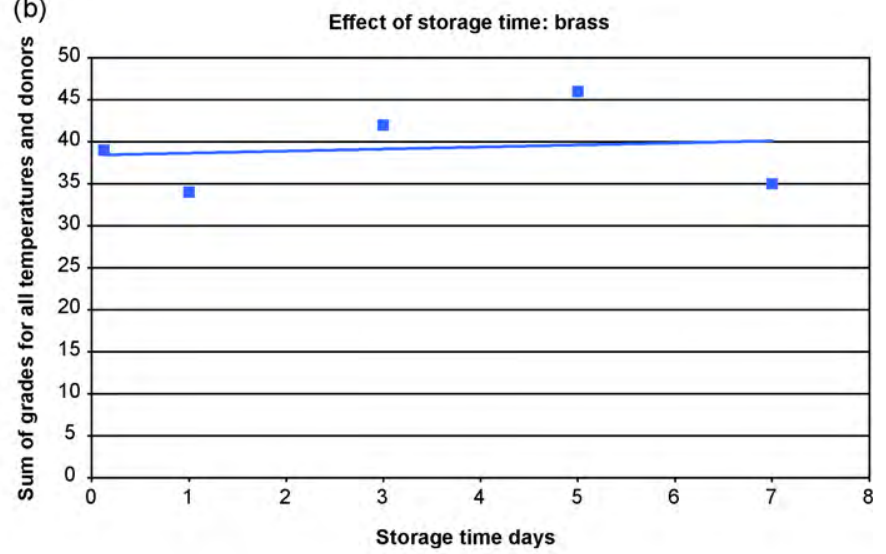

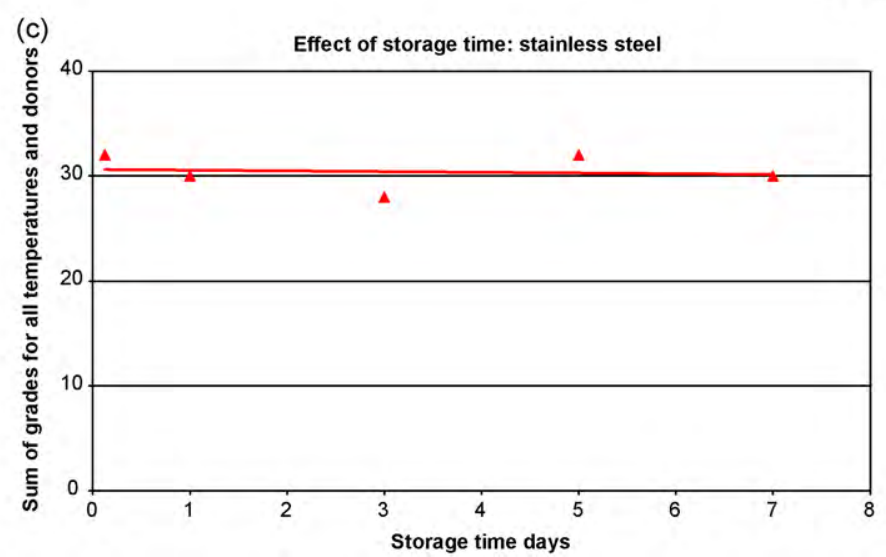

Fig. 1. (a) Effect of storage time on aluminium, (b) brass, and (c) stainless steel.

after $3 \mathrm{~h}$ storage. Similarly, donors 1 and 2 gave poor marks on aluminium on the 3-day sample, but these were not enhanced at higher temperature and this is consistent with the observations that marks tended to degrade at higher temperatures on aluminium. It is not known why these two data points on day 3 are lower and it may be due to differences in secretions. The poor results for donors 1 and 2 on brass after $3 \mathrm{~h}$ storage might suggest that a slow chemical reaction is occurring (e.g. corrosion) that is needed in order to initiate the reaction occurring on heating, but then donor 3's marks would also be expected to be poorer. An alternative explanation might be that on these 2 days the secretions from these two donors were significantly different from other days.

\subsection{Effect of storage time}

Fig. 1a-c shows the total of the grades for all three donors treated at all six temperatures, for metal discs heat-treated after different elapsed time periods up to a week. Individual values are given in Table 4. Fig. 1a shows some variation in the results for

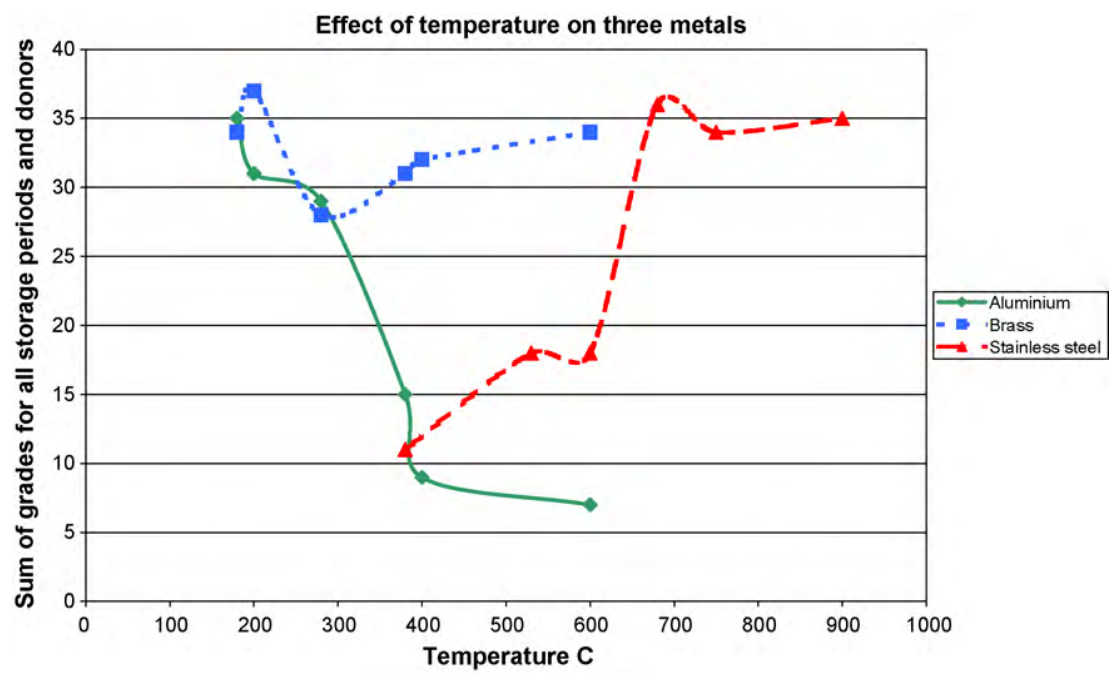

Fig. 2. Effect of temperature on three metals. 
Table 2

Grading score for individual donors for 30 prints over six temperatures and five storage times.

\begin{tabular}{lccc}
\hline & Donor 1 & Donor 2 & Donor 3 \\
\hline Brass & 61 & 53 & 83 \\
Aluminium & 37 & 32 & 57 \\
Stainless steel & 42 & 42 & 68 \\
Total & 140 & 127 & 208 \\
\hline
\end{tabular}

Score range for each metal and donor: 0-90. Total range for each donor: 0-270.

aluminium, which appears to show a dip at 3 days due to the poor marks obtained from donors 1 and 2 , as discussed above. Donor 3 gave consistent marks over all storage periods below $400{ }^{\circ} \mathrm{C}$, although results were more variable at $400{ }^{\circ} \mathrm{C}$ and $600{ }^{\circ} \mathrm{C}$. A trend line for aluminium also shows little dependence on time. Brass also showed some variation (34-46) but a trend line for these data points is horizontal (Fig. 1b), suggesting there is no dependence on storage time. Stainless steel showed very little variation with a total grade score of 28-32 out of a possible 54 .

These observations are consistent with Bond's [1] observations on elapsed time, and the ability to recover marks after a period of time is a useful benefit of the technique, although the present study has only looked at elapsed periods of up to 7 days.

\subsection{Effect of temperature}

Fig. 2 shows the effect of temperature on each of the three metals. As previously noted, the data are summations from three donors and five time periods so the maximum possible sum of grades for each temperature is 45 . Temperature can have a dramatic effect on fingermark recovery and aluminium clearly shows a decline at temperatures above $280{ }^{\circ} \mathrm{C}$. In the case of brass there is an apparent decline between $200{ }^{\circ} \mathrm{C}$ and $280{ }^{\circ} \mathrm{C}$ from a score of 37 to 28 , and this may be due to differential oxidation rates. On the other hand, stainless steel shows a clear increase when heated above $600{ }^{\circ} \mathrm{C}$. The causes of these trends are discussed further in Section 4.2. These findings are important if marks are to be effectively recovered through a heating process, and will help define whether marks are likely to be recovered after a fire.

\subsection{Effect of reheating after initial recovery}

The samples were treated in two separate phases and, in each phase, the disc was heated at the lowest temperature (e.g. $200{ }^{\circ} \mathrm{C}$ ) then photographed and reheated (e.g. $400{ }^{\circ} \mathrm{C}$ ) before a third and final cycle (e.g. $600{ }^{\circ} \mathrm{C}$ ). An important observation from this was that a poor mark could sometimes be enhanced by further heating. In the case of aluminium reheating the metal usually degraded the mark and in the case of stainless steel reheating usually enhanced

Table 3

Number of prints recovered at different grades.

\begin{tabular}{|c|c|c|c|c|c|c|c|c|c|}
\hline Metal & Brass & Brass & Brass & Aluminium & Aluminium & Aluminium & Stainless steel & Stainless steel & Stainless steel \\
\hline Donor & 1 & 2 & 3 & 1 & 2 & 3 & 1 & 2 & 3 \\
\hline Grade 3 & 12 & 7 & 24 & 5 & 3 & 12 & 2 & 3 & 13 \\
\hline Grade 2 & 8 & 13 & 5 & 8 & 7 & 9 & 13 & 12 & 12 \\
\hline Grade 1 & 9 & 6 & 1 & 6 & 9 & 3 & 10 & 9 & 5 \\
\hline Grade 0 & 1 & 4 & 0 & 11 & 11 & 6 & 5 & 6 & 0 \\
\hline
\end{tabular}

Score range 0-30 (six temperatures and five time intervals).

Table 4

Total score of fingerprint quality from three donors.

\begin{tabular}{|c|c|c|c|c|c|c|c|}
\hline \multirow[t]{2}{*}{ Metal } & \multirow[t]{2}{*}{ Temp $\left({ }^{\circ} \mathrm{C}\right)$} & \multicolumn{5}{|c|}{ Time (days) } & \multirow[t]{2}{*}{ Total } \\
\hline & & 0.125 & 1 & 3 & 5 & 7 & \\
\hline Brass & 200 & 4 & 7 & 9 & 9 & 8 & 37 \\
\hline Brass & 400 & 9 & 5 & 7 & 6 & 5 & 32 \\
\hline Brass & 600 & 9 & 6 & 6 & 7 & 6 & 34 \\
\hline Brass & 180 & 4 & 8 & 8 & 9 & 5 & 34 \\
\hline Brass & 280 & 4 & 6 & 5 & 9 & 4 & 28 \\
\hline Brass & 380 & 9 & 2 & 7 & 6 & 7 & 31 \\
\hline Brass & Sum & 39 & 34 & 42 & 46 & 35 & 196 \\
\hline Aluminium & 200 & 9 & 8 & 3 & 6 & 5 & 31 \\
\hline Aluminium & 400 & 2 & 0 & 2 & 3 & 2 & 9 \\
\hline Aluminium & 600 & 0 & 1 & 0 & 5 & 1 & 7 \\
\hline Aluminium & 180 & 8 & 9 & 3 & 8 & 7 & 35 \\
\hline Aluminium & 280 & 5 & 7 & 4 & 6 & 7 & 29 \\
\hline Aluminium & 380 & 1 & 2 & 3 & 6 & 3 & 15 \\
\hline Aluminium & Sum & 25 & 27 & 15 & 34 & 25 & 126 \\
\hline Stainless steel & 600 & 5 & 5 & 2 & 3 & 3 & 18 \\
\hline Stainless steel & 750 & 7 & 6 & 7 & 7 & 7 & 34 \\
\hline Stainless steel & 900 & 8 & 6 & 7 & 7 & 7 & 35 \\
\hline Stainless steel & 380 & 2 & 1 & 2 & 4 & 2 & 11 \\
\hline Stainless steel & 530 & 3 & 4 & 3 & 4 & 4 & 18 \\
\hline Stainless steel & 680 & 7 & 8 & 7 & 7 & 7 & 36 \\
\hline Stainless steel & Sum & 32 & 30 & 28 & 32 & 30 & 152 \\
\hline
\end{tabular}

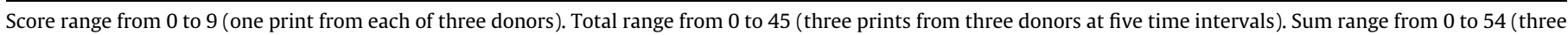
prints on each of three metals at six temperatures). 
Table 5

Effect of reheating on print recovery.

\begin{tabular}{llll}
\hline Metal & Time (days) & Temp $\left({ }^{\circ} \mathrm{C}\right)$ & Print grade for donor 1 \\
\hline Brass & 1 & 200 & 2 \\
Brass & 1 & 400 & 1 \\
Brass & 1 & 600 & 2 \\
Brass & 5 & 200 & 3 \\
Brass & 5 & 400 & 1 \\
Brass & 5 & 600 & 2 \\
Brass & 7 & 200 & 3 \\
Brass & 7 & 400 & 1 \\
Brass & 7 & 600 & 2 \\
\hline
\end{tabular}

the mark, although degradation sometimes began to occur at $900{ }^{\circ} \mathrm{C}$. It was observed that the colour of the surface of stainless steel changed and this is a factor contributing to the enhancement. With brass, however, an apparent degradation of the mark and subsequent recovery at higher temperature was sometimes observed. This was only observed in brass and examples are shown in Table 5 . These observations are discussed further in Section 4.2 .

\subsection{Effect of metal substrate}

As can be seen from the previous discussion, the metal substrate is important. Heating samples of brass gave good enhancement (total score of 196 out of 270) and heating stainless steel gave reasonable enhancement (152 out of 270) but aluminium was poorest (126 out of 270 ), as seen in Tables 3 and 4. Fig. 2 also shows that metals behave differently in different temperature regimes: brass appeared not to show much variation under the conditions studied (although sometimes the mark appeared to degrade and then improve); aluminium degraded when heated above $280{ }^{\circ} \mathrm{C}$; and, stainless steel gave poor recovery until heated above $600{ }^{\circ} \mathrm{C}$.

\subsection{Microscopic examination}

The discs were examined optically under a stereomicroscope, with objective lenses ranging from $5 \times$ to $40 \times$. Ridge marks on the brass showed surface discolouration that sometimes had a dendritic appearance. Pits were observed within these regions, as seen in Fig. 3b and this might be suggestive of chloride attack, as proposed by Bond [1]. However, an explanation is then needed for the brighter regions seen around the pits, and it is suggested that this may be differential growth of the oxide layer.

Stainless steel showed similar brighter regions along the ridge pattern with bright spots, but no pits were observed in any sample. Aluminium showed a few bright spots but no pitting and no continuous surface change along ridges. Chloride is known to cause pitting in metals, but little evidence was seen apart from possible pitting in brass.

\subsection{Heating of metal with laboratory-grade chemicals}

A disc of brass and a disc of stainless steel were heated sequentially to $750{ }^{\circ} \mathrm{C}$. At $600{ }^{\circ} \mathrm{C}$ stainless steel produced interference colours whereas brass remained golden until $750{ }^{\circ} \mathrm{C}$ when it went silver, probably due to zinc oxidation. In both cases the control fingermarks developed during the heating process in the same way as they had done in the other series of tests.

Bond [1] suggests chloride corrosion as contributing to thermal fingermark development. In the present study no corrosion products were observed with salt solution except when heated above $600{ }^{\circ} \mathrm{C}$ where a slight colour difference was noted in areas that had previously been covered by solution but were free from (a)

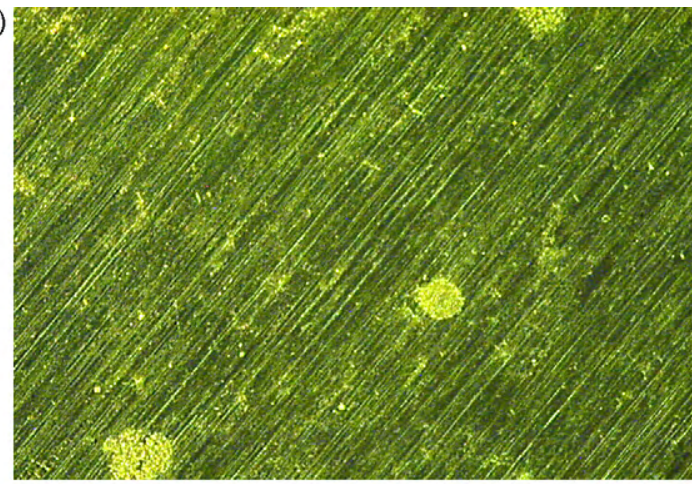

(b)

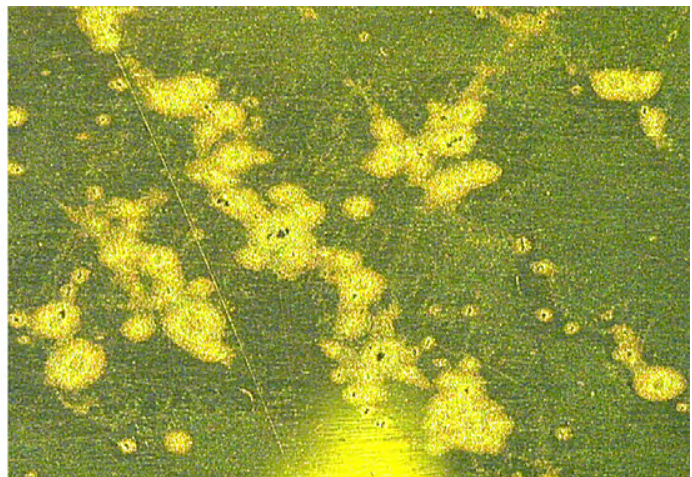

(c)

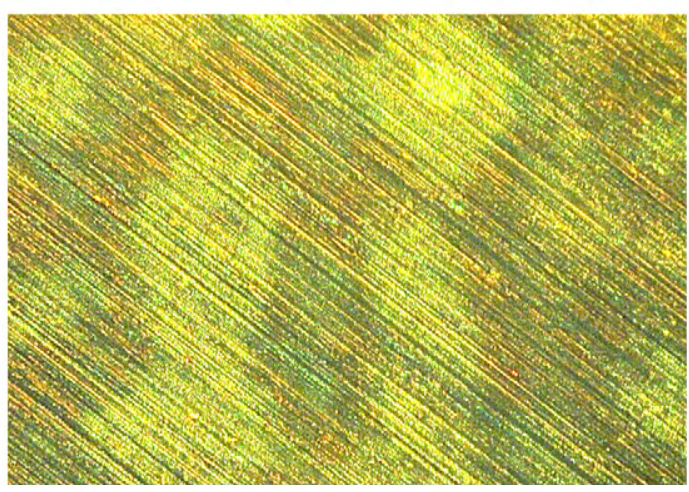

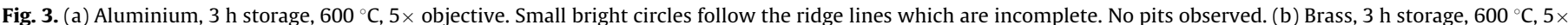

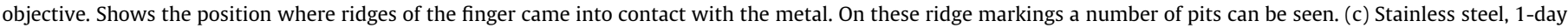
storage, $950{ }^{\circ} \mathrm{C}, 5 \times$ objective. Shows ridge contact with small bright circles, but no pits observed.

Please cite this article in press as: G. Wightman, D. O'Connor, The thermal visualisation of latent fingermarks on metallic surfaces, Forensic Sci. Int. (2010), doi:10.1016/j.forsciint.2010.05.007 
Free energy of chloride formation

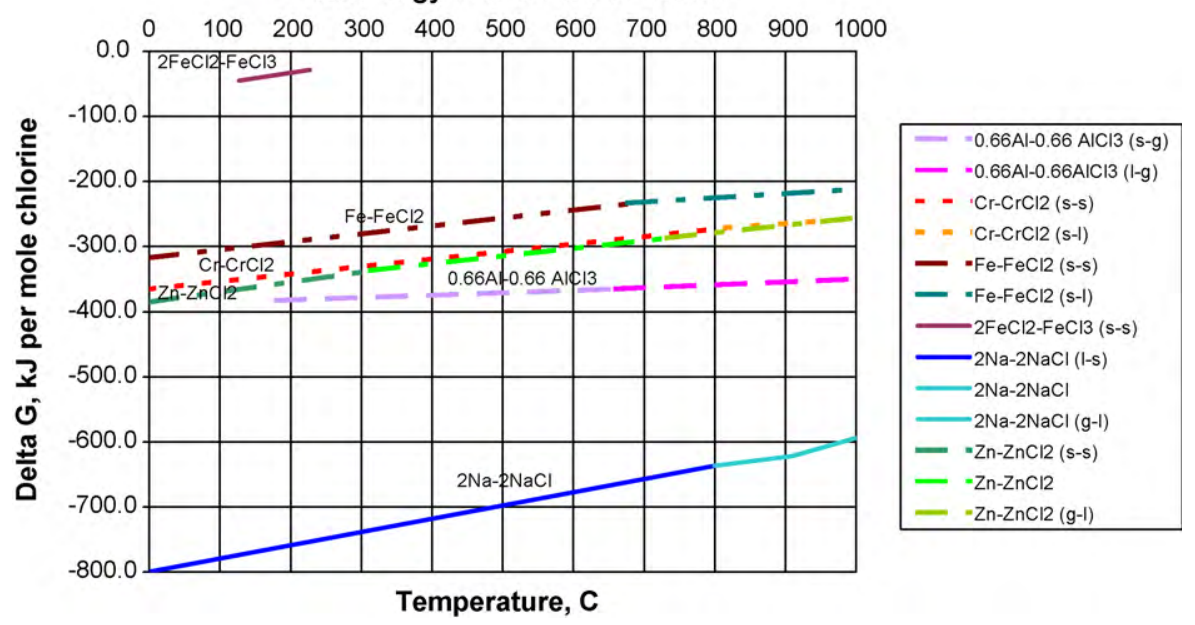

Fig. 4. Free energy of chloride formation.

salt crystals. Similarly, anhydrous salt crystals showed little effect except for limited corrosion of stainless steel when heated to $750{ }^{\circ} \mathrm{C}$. On brass at $750{ }^{\circ} \mathrm{C}$, the area around the salt crystals remained a gold colour whereas the rest of the brass turned silver, but no evidence of pitting was observed. In contrast, pitting was observed from the fingermark on brass.

To investigate the alternative hypothesis whether oxide reduction may be occurring, various organic materials were also heated with the metal samples. Graphite and paraffin showed no obvious change at any temperature on either metal. Oleic acid initially evaporated and discoloured the surrounding metal, possibly by inhibiting the rate of oxide formation and allowing interference colours to develop. At higher temperature, the oleic acid charred and this discoloured area continued to be differentiated in appearance from the rest of the metal.

The effect of salt crystals at $750{ }^{\circ} \mathrm{C}$ on brass and the effect of oleic acid on both brass and stainless steel had a similar appearance to the effect of vapours such as iodine or ammonium sulphide on metals where chemical reaction occurs [5]. In the present study, however, it may be that the vapour is inhibiting oxygen access and hence reducing the oxidation taking place. The absence of corrosion products and pitting with these materials raises a question as to whether a corrosion mechanism is causing fingermark enhancement, and further testwork is needed.

\section{Metallurgical considerations}

\subsection{Reaction of chlorides}

Bond [1] developed a theory to explain the enhancement of fingermarks on metals based on electrochemical reactions in the presence of the salt residue from sweat. However, this model may not be applicable at higher temperatures. An electrochemical reaction can occur in the presence of halide ions and water, but if this is the mechanism, then evidence of marks might be expected to be present without enhancement. On heating, the water would evaporate and the only mechanism for ion movement would be solid state diffusion (which is normally a relatively slow reaction) or molten salt corrosion.

Free energy diagrams have been produced using data from Kubaschewski and Alcock [6]. One or two data points differ slightly from other published diagrams but none of the differences is sufficient to affect the following discussion. Examination of the chloride free energy diagram (Fig. 4) shows that sodium chloride is much more stable than the other metal chlorides under consideration and a simple displacement reaction of sodium ions by other cations to form a salt should not occur. Metathesis reaction between an oxide layer and the chloride is an alternative reaction. However, the free energy diagrams Figs. 4 and 5 suggest that the

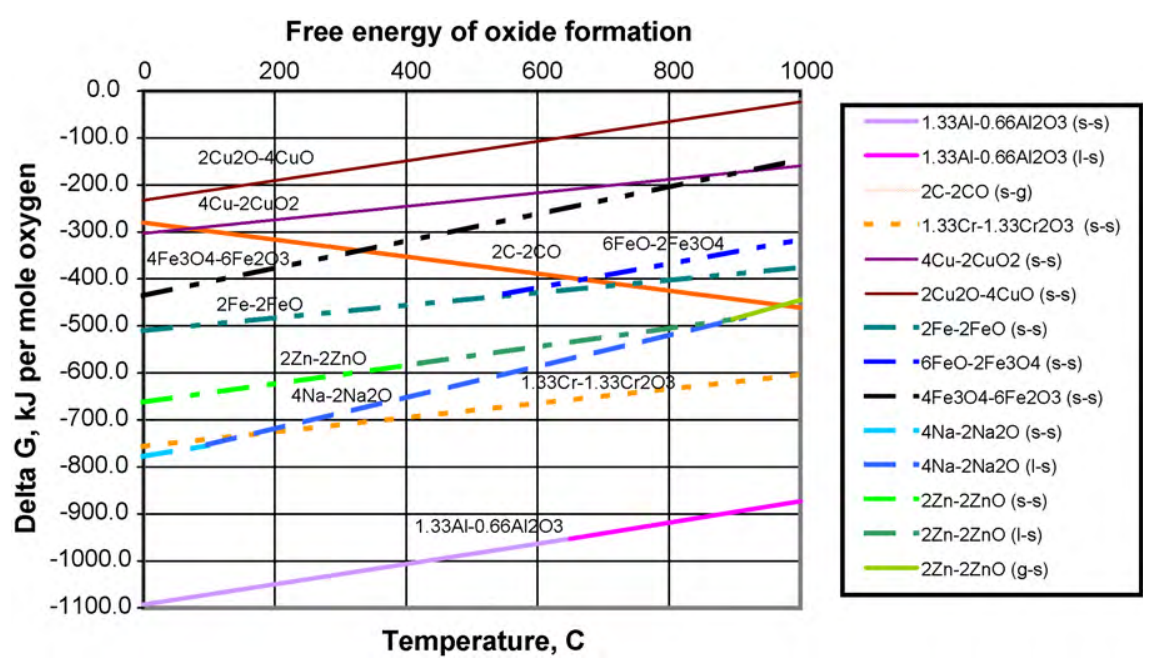

Fig. 5. Free energy of oxide formation. 
free energy difference between sodium chloride and other chlorides is much greater than between sodium oxide and other oxides. Thus reactions such as:

(a) $\mathrm{Fe}+3 \mathrm{NaCl} \rightarrow \mathrm{FeCl}_{3}+3 \mathrm{Na}$, or

(b) $\mathrm{ZnO}+2 \mathrm{NaCl} \rightarrow \mathrm{ZnCl}_{2}+\mathrm{Na}_{2} \mathrm{O}$

are thermodynamically unfavourable, and an alternative mechanism is required.

On observing the heated discs it is apparent that an oxide film is forming and is producing interference colours. It is therefore proposed that it is the growth of this film that enhances the mark due to differential oxidation where the ridges have contacted the metal, and the enhanced contrast caused by the interference colours generated by the film. This mechanism would explain: the temperature effects observed; the ability sometimes to improve contrast by reheating the sample; and is consistent with the apparent absence of chloride corrosion in aluminium and stainless steel.

\subsection{Oxide formation}

Fig. 5 is a free energy diagram for the formation of oxides, and shows that thermodynamically carbon could reduce copper, iron and zinc oxides, three of the oxides involved in this study. Aluminium and chromium lines do not cross the carbon line within the temperature range of this study, although chromium could be reduced at non-unit activities. One possibility that therefore needs to be considered is that organic secretions in the sweat may be chemically reducing oxide as it forms. An alternative explanation is that organic secretions or salt are acting as a barrier to oxidation.

\subsubsection{Aluminium oxidation}

Aluminium is a reactive metal but it is protected by its oxide layer since it is a good electrical and ionic insulator. Fig. 5 shows that the carbon line and aluminium line do not cross within the temperature range examined, and hence carbon cannot reduce aluminium oxide. Organic material in sweat is therefore unlikely to reduce the oxide. The growth of the oxide film itself is limited on heating until the aluminium reaches the liquid state (above $660{ }^{\circ} \mathrm{C}$ ) when the oxide film is able to move and bare metal is exposed [7]. Akagwu et al. [8] also studied the oxidation of molten aluminium and found the oxide thickness was less than $250 \mathrm{~nm}$ after $30 \mathrm{~min}$ at $950{ }^{\circ} \mathrm{C}$. Oxide thickness after $5 \mathrm{~min}$ heating at temperatures up to $600{ }^{\circ} \mathrm{C}$ will therefore be considerably less than this and hence will be too thin to produce interference colours. Consequently, significant mark enhancement on aluminium is not expected to occur using this technique, although it might be possible to anodise the aluminium to produce thicker oxide films.

\subsubsection{Stainless steel oxidation}

Stainless steel is protected from corrosion by a chromium oxide layer. Some grades of stainless steel can be susceptible to chloride attack and pitting, but pitting was not observed in the present study apart from some spots on fingermarks when heated at $900{ }^{\circ} \mathrm{C}$. Interference colours were generated on heating due to oxide growth. Interference occurs when light of a particular wavelength in the white light spectrum is reflected from two surfaces a slight distance apart (e.g. oxide and metal). In reflectance mode extinction occurs when this separation is a quarter wavelength and the complimentary colour is seen. Higher order interference fringes can also occur but may be fainter due to absorption by the thicker layer. Michel-Levy [9] produced a chart of interference colours produced by birefringence interference and this chart can be used to compare with the colours produced by reflection interference.

Stainless steel showed little colouration below $600{ }^{\circ} \mathrm{C}$. When heated for $5 \mathrm{~min}$ at $600{ }^{\circ} \mathrm{C}$ the metal became an orange-brown background with uncoloured regions on the mark. At higher temperatures these shades changed, and at $900{ }^{\circ} \mathrm{C}$ the background was blue with areas of the mark orange-brown. Comparing this with the colours on the Michel-Levy chart the orange colour at $600{ }^{\circ} \mathrm{C}$ could correspond to $450 \mathrm{~nm}$ and an oxide thickness of $110 \mathrm{~nm}$, with the $900{ }^{\circ} \mathrm{C}$ colours corresponding to an oxide thickness of $160 \mathrm{~nm}$ for blue and $110 \mathrm{~nm}$ for orange. If secretions in the fingermark are inhibiting oxidation, then these differences in colour (and hence contrast) would be seen.

\subsubsection{Brass oxidation}

In the case of brass, fingermarks appear to be enhanced in the same way as for stainless steel, although interference colours are not observed. Brass is protected by preferential oxidation of the zinc at the surface leading to slight dezincification. Zinc oxide then protects the metal in the same way as chromium oxide in stainless steel and aluminium oxide on aluminium. However, the electronic and ionic conductivity of zinc oxide and chromium oxide are higher than aluminium oxide due to lattice imperfections and oxide growth can occur on heating. In the case of brass, the full range of interference colours is not observed because the brass is coloured and is absorbing part of the spectrum. However, where finger secretions are present contrast can still occur due to differences in the oxide film thickness producing extinction, but this extinction will be seen as shades of light and dark rather than different colours. In practice very good contrast was observed in most cases with various gradations of shade producing a 3D impression. However, because the contrast on brass is due on a difference of intensity, it is possible for the contrast to be reduced when both the mark and background are undergoing partial extinction but of different orders. As further oxidation occurs (for example, by treatment at a higher temperature in the current testwork) the oxide continues to grow at different rates and can again reach a condition where one region of the mark undergoes extinction whilst the other does not. Table 6 gives an example how differential growth might cause contrast to change on heating whilst Fig. 6 shows examples of the contrast obtained with the three metals used in this study.

\section{Future work}

In order to confirm that differential oxidation is occurring, the mark regions need more detailed microanalysis by SEM and EDX or possibly by AFM. In the present study oxidation was controlled by changing the temperature, but the rate of oxidation could also be

Table 6

Example of possible contrast with differential oxide growth

\begin{tabular}{|c|c|c|c|c|}
\hline Thickness of first region & Interference in first region & Thickness of second region & Interference in second region & Contrast \\
\hline $250 \mathrm{~nm}$ & Constructive & $125 \mathrm{~nm}$ & Destructive & Good \\
\hline $500 \mathrm{~nm}$ & Constructive & $250 \mathrm{~nm}$ & Constructive & Poor \\
\hline $750 \mathrm{~nm}$ & Constructive & $375 \mathrm{~nm}$ & Destructive & Good \\
\hline
\end{tabular}

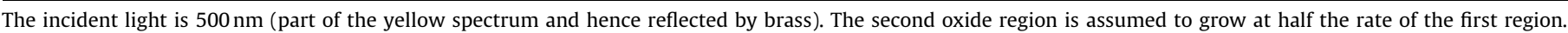
Destructive interference occurs when thickness $=(2 n+1) \lambda / 4$. Constructive interference occurs when thickness $=n \lambda / 2$. 
(a)

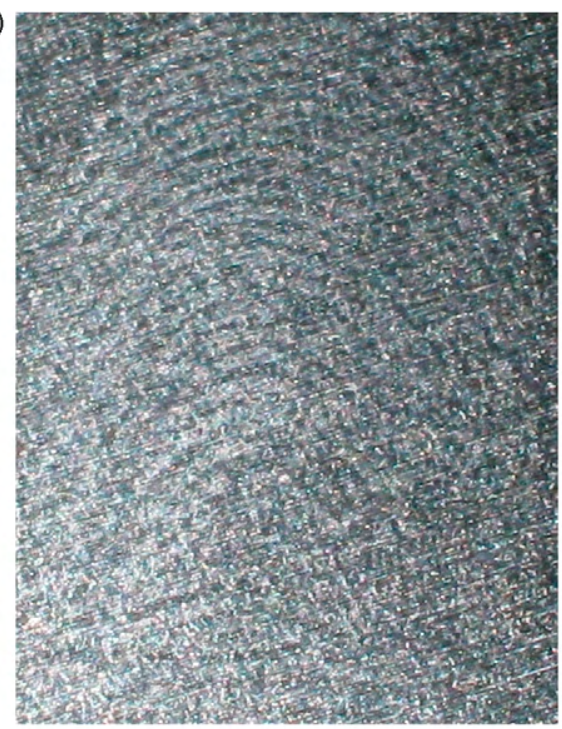

(c)

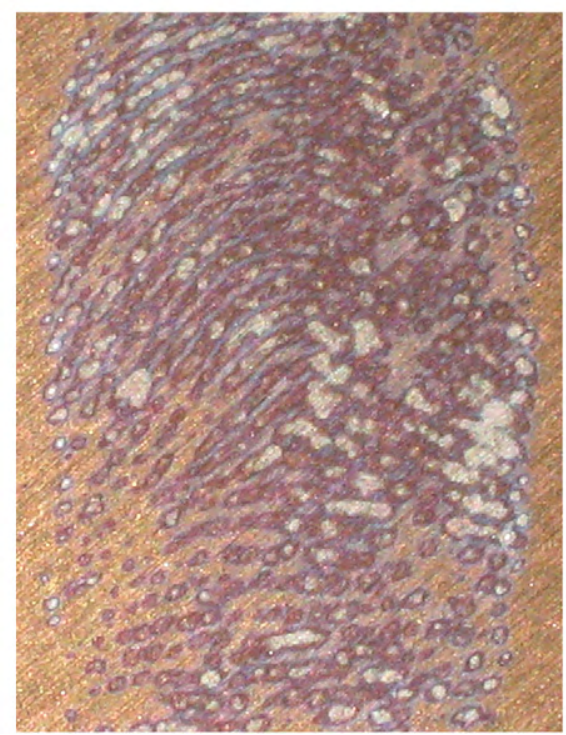

(b)

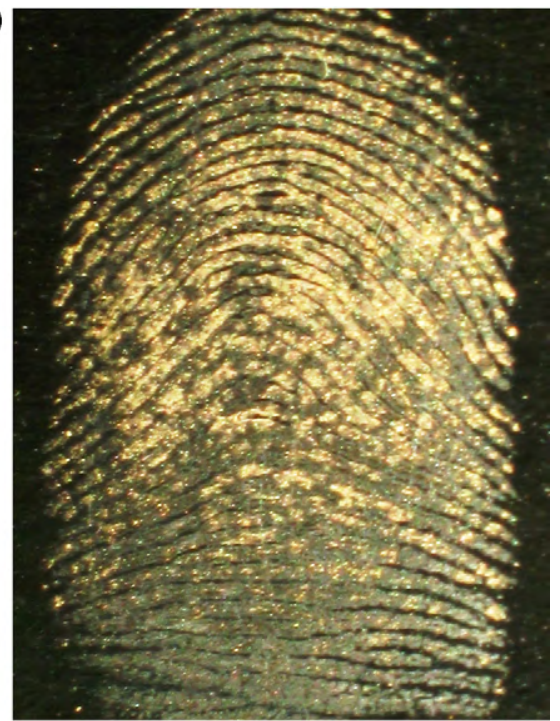

(d)

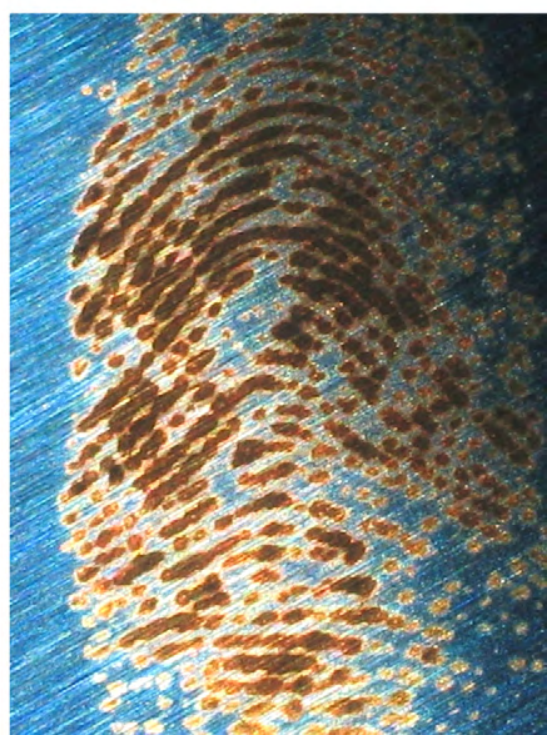

Fig. 6. Contrast on (a) aluminium 3 days storage, $180^{\circ} \mathrm{C}$; (b) brass 3 days storage, $180^{\circ} \mathrm{C}$; (c) stainless steel 3 days storage, $680^{\circ} \mathrm{C}$; (d) stainless steel 3 days storage, $900{ }^{\circ} \mathrm{C}$.

controlled by employing different times for oxidation or by varying the partial pressure of oxygen in the furnace. The component of the sweat secretion that is inhibiting oxidation needs to be confirmed by microanalysis and by further comparison with pure compounds. Another area for potential study is whether marks from different donors on a single source (e.g. a metal door handle) can be separated by controlling the oxidation rate e.g. by a series of sequential partial oxidations.

\section{Conclusion}

A study has been carried out on the recovery of latent fingermarks on metallic surfaces by heating the sample for $5 \mathrm{~min}$ in a muffle furnace at various temperatures, and the protocol adopted proved very effective at visualising latent marks. Whilst there were variations between the donors, the recovery ranged from $47 \%$ to $77 \%$ of the maximum recovery for the three donors based on a quality grading scheme of $0-3$ for individual marks. The summation of these grades over a range of different variables allowed factors affecting the mark quality to be identified, and storage time of the samples appeared to have little effect up to the 7-day time-scale that was studied. However, the temperature of heating had an important effect and was dependent on the metal. Brass appeared to be the least dependent on temperature up to $600{ }^{\circ} \mathrm{C}$. Aluminium gave poor visualisation when heated above $280^{\circ} \mathrm{C}$, whereas stainless steel only gave good visualisation when heated above $600{ }^{\circ} \mathrm{C}$ but began to lose detail when heated to $900{ }^{\circ} \mathrm{C}$.

It was observed that samples can sometimes be reheated to further enhance recovery although care is needed so that the mark is not permanently degraded by over-oxidation. This ability to retreat the mark could be important from a forensic perspective and further work is required on the effect of time, temperature and oxygen partial pressure on fingermark recovery.

Some evidence of pitting was observed in brass but not in aluminium or stainless steel. It was also observed that interference colours form due to the oxide layer, and it is postulated that enhancement occurs due to differential oxide growth producing contrasting interference colours, rather than by a corrosion mechanism. 


\section{References}

[1] J.W. Bond, Visualisation of latent fingerprint corrosion of metallic surfaces, J. Forensic Sci. 53 (4) (2008) 812-822.

[2] J.W. Bond, The thermodynamics of latent fingerprint corrosion of metal elements and alloys, J. Forensic Sci. 53 (6) (2008) 1344-1352.

[3] J.W. Bond, On the electrical characteristics of latent finger mark corrosion of brass, J. Phys. D: Appl. Phys. 41 (2008) 125502 (10 pp.).

[4] H.H. Strehblow, Mechanisms of pitting corrosion, in: P. Marcus (Ed.), Corrosion Mechanisms in Theory and Practice, 2nd edition, Marcel Dekker AG, New York, 2002
[5] J.P. Chilton, Principles of Metallic Corrosion, Royal Institute of Chemistry Monograph, W Heffer and Sons, Cambridge, 1969.

[6] O. Kubaschewski, C.B. Alcock, Metallurgical Thermochemistry, Pergamon Press, Oxford, 1979.

[7] G. Wightman, D.J. Fray, The dynamic oxidation of aluminium and its alloys, Met. Trans. B 14B (December) (1983) 625-631.

[8] I. Akagwu, R. Brooks, Z. Fan, B. Ralph, P. Quested, Liquid state oxidation of aluminium, in: 2nd Materials Research Conference for Young Researchers, 1718th February, IOM3, London, 2003.

[9] A. Michel-Levy, Les mineraux des roches, Librairie Polytechnique, Paris, 1888.

Please cite this article in press as: G. Wightman, D. O'Connor, The thermal visualisation of latent fingermarks on metallic surfaces, Forensic Sci. Int. (2010), doi:10.1016/j.forsciint.2010.05.007 